\title{
The Localization of Phenolic Compounds in Liposomal Bilayers and Their Effects on Surface Characteristics and Colloidal Stability
}

\author{
Swapnil A. Malekar, ${ }^{1}$ Ashish L. Sarode, ${ }^{1,2}$ Alvin C. BachII, ${ }^{1}$ and David R. Worthen ${ }^{1,2,3}$
}

Received 28 October 2015; accepted 13 January 2016; published online 2 February 2016

\begin{abstract}
The interactions with and effects of five chemically distinct, bioactive phenolic compounds on the lipid bilayers of model dipalmitoylphosphatidylcholine (DPPC) liposomes were investigated. Complementary analytical techniques, including differential scanning calorimetry (DSC) and phosphorus and proton nuclear magnetic resonance spectroscopy (NMR), were employed in order to determine the location of the compounds within the bilayer and to correlate location with their effects on bilayer characteristics and liposomal stability. As compared to the phenolic compounds localized in the glycerol region of the DPPC head group within the bilayer, which enhanced the colloidal stability of the liposomes, compounds located closer to the center of the bilayer reduced vesicle stability as a function of time. Molecules present in the upper region of liposomal DPPC acyl chains $\left(\mathrm{C}_{1}-\mathrm{C}_{10}\right)$ inhibited liposomal aggregation and size increase, perhaps due to tighter packing of adjoining DPPC molecules and increased surface exposure of DPPC phosphate head groups. These data may be useful for designing liposomal systems containing hydrophobic phenols and other small molecules, selecting appropriate analytical methods for determining their location within liposomal bilayers, and predicting their effects on liposome characteristics early in the liposome formulation development process.
\end{abstract}

KEY WORDS: bilayer; liposome; phenolic; stability; surface characterization; vesicle.

\section{INTRODUCTION}

Liposomes are employed in a variety of applications, including pharmaceuticals, foods, cosmetics, diagnostics, and bioanalysis (1). Liposomes, consisting of one or more lipid bilayers that encapsulate aqueous phases, have been categorized as small unilamellar vesicles $(20-100 \mathrm{~nm})$, large unilamellar vesicles $(50-400 \mathrm{~nm})$, and multilamellar vesicles (400-5000 nm) (2). Liposomes composed of phospholipids such as dipalmitoylphosphatidylcholine (DPPC) tend to be inert, non-immunogenic, and minimally toxic and may be particularly attractive for the encapsulation and delivery of bioactive compounds to living systems (3). Possessing both hydrophobic and hydrophilic domains comprising a lipid bilayer and an aqueous core, respectively, liposomes have the capacity to encapsulate drugs, biologics, and other chemical substances with diverse physicochemical properties (4). While the aqueous regions typically encapsulate hydrophilic

Electronic supplementary material The online version of this article (doi:10.1208/s12249-016-0483-5) contains supplementary material, which is available to authorized users.

$\overline{{ }^{1} \text { Department of Biomedical and Pharmaceutical Sciences, College of }}$ Pharmacy, University of Rhode Island, 495M Pharmacy Building, 7 Greenhouse Drive, Kingston, Rhode Island 02881, USA.

${ }^{2}$ Department of Chemical Engineering, College of Engineering, University of Rhode Island, Kingston, Rhode Island 02881, USA.

${ }^{3}$ To whom correspondence should be addressed. (e-mail: drworthen@uri.edu) compounds, hydrophobic substances tend to localize in the lipid bilayers. Accordingly, liposomes may be employed for the formulation and delivery of diverse compounds, such as hydrophilic and hydrophobic drugs and natural products without the extensive use of additional organic solvents or solubilizing agents (5). However, the incorporation of such compounds in liposomes may strongly influence their physicochemical and surface characteristics and colloidal stability, considerations that are of particular importance in the design of liposomal formulations.

The effect of the location and orientation of amphiphilic polymers, such as poloxamers, in lipid vesicles, and their interactions with the lipid vesicle bilayer on the colloidal stability of the vesicles, has been reported (6). The insertion of the hydrophobic portion of the polymer inside the bilayer and localization of the hydrophilic portion on the vesicular surface may impart steric hindrance at the interface, thereby improving the colloidal stability of lipid vesicles in bulk solvent. The incorporation of small molecules in liposomes may also have anecdotal effects on membrane dynamics and the phase behavior of liposomal bilayers, as described both experimentally and by molecular simulation (7-11). The interaction of hydrophobic compounds with lipid molecules and their location and orientation in lipid bilayers may affect bilayer properties in different ways. For example, it was suggested that the phytoestrogen genistein increased the ordering of lipid acyl chains, thereby increasing lipid bilayer rigidity (12). Such changes in lipid bilayer properties as a result of the incorporation of hydrophobic molecules therein may impact 
the surface characteristics and colloidal stability of liposomes, although not necessarily in predictable ways.

A number of natural and synthetic hydrophobic phenolics exert useful biological, therapeutic, and nutritional effects in living systems. Likewise, many of these compounds have been employed as antioxidants and preservatives in pharmaceutical, cosmetic, and nutritional products. However, the use of such compounds in these applications is often compromised due to poor solubility, chemical instability, and challenges in targeted delivery to humans and animals. Likewise, certain bioactive polyphenols may interact with lipid membranes and alter membrane fluidity, thermal properties, and their capacity to stably encapsulate small molecules. Tannic acid, a plantderived polyphenol, may induce interdigitation of DPPC residues in liposomal systems while collapsing the interbilayer fluid spaces in DPPC liposomes (13). A polyphenolic stilbene that occurs in a number of foods, trans-resveratrol induces a substantial increase in the mean molecular area of dipalmitoylphosphatidyl ethanolamine lipid monolayers (14). The localization of small molecule polyphenols in lipid membranes appears to be highly dependent upon the type of lipid comprising the membranes, with resveratrol inserting more deeply into cationic lipid membranes than into zwitterionic lipid membranes (15). Evidently, the discrete effects of polyphenolic compounds on lipid membranes and their location therein are important considerations in the formulation of polyphenol-containing liposomes. Strong interest exists in the characterization of polyphenolic interactions with liposomes in order to facilitate the development of stable liposomal formulations and delivery systems for biologically active phenolics, since liposomes may serve as attractive delivery systems for these compounds.

The purpose of this work was to determine the physiochemical and thermodynamic changes in lipid bilayer characteristics upon the incorporation of a series of chemically diverse, biologically active phenolic compounds into model DPPC liposomes, the specific interactions of the compounds with DPPC molecules, their localization in the lipid bilayer, and their subsequent effects on liposomal stability. No study in the literature combines DSC and surface charge analysis with both ${ }^{1} \mathrm{H}$ - and ${ }^{31} \mathrm{P}-\mathrm{NMR}$ in order to investigate in detail the membrane localization of polyphenolics and their subsequent effects on liposomal stability. It was hypothesized that the surface properties and colloidal stability of model DPPC liposomes might be significantly altered due to polyphenolinduced changes in bilayer properties and that a correlation between membrane localization of the polyphenolics and their effects on liposome characteristics and stability could be developed. Accordingly, the biologically active, hydrophobic phenolics raloxifene (RAL), garcinol (GAR), quercetin (QTN), trans-resveratrol (RVR), and bisphenol A (BPA) were therefore examined. RAL is a diphenolic, selective estrogen receptor modulator. GAR is a polyisoprenylated benzophenone known for its antibiotic, antiviral, antifungal, and anticancer activities $(16,17)$. QTN and RVR are plant-derived phenolics used for a variety of ailments such as inflammation, viral diseases, asthma, eczema, and cancer (18-23). RVR is a polyphenolic phytoalexin and a derivative of stilbene, while QTN is a heterocyclic flavanol, both of which have low aqueous solubility. BPA is a potentially toxic compound reported to cause behavioral alterations and preneoplastic lesions in the prostate, mammary gland, and uterus (23). BPA, often studied in but challenging to administer to animals during toxicity studies, is thus a candidate for in vivo delivery via liposomes. Accordingly, BPA was examined here not as a therapeutic agent but in order to appreciate its interaction with DPPC bilayers owing to its hydrophobicity, aromatic, diphenolic character, and transoid conformation due to the presence of two geminal methyl groups.

\section{MATERIALS AND METHODS}

\section{Materials}

1,2-Dipalmitoyl-sn-glycero-3-phospocholine monohydrate (DPPC) was purchased from Corden Pharma (CO, USA), stored at $-20^{\circ} \mathrm{C}$ until use, and used within 1 week of receipt from the manufacturer. QTN was purchased from Acros Organics (NJ, USA), RAL from Fisher Scientific (Pittsburgh, PA), RVR from CS Inc. (Danbury, CT, USA), GAR from Enzo Life Sciences, (NY, USA), and BPA from SigmaAldrich (St. Louis, MO, USA). Phosphate-buffered saline (PBS) tablets were purchased from MP Biomedicals (Solon, OH, USA). All other reagents were purchased from Fisher Scientific and were of analytical grade.

\section{Liposome Preparation}

Vesicles were prepared at a $17 \mathrm{mM}$ lipid (DPPC)/2 mM phenolic concentration for all formulations. Blank DPPC control vesicles contained only DPPC. For the ${ }^{31} \mathrm{P}-\mathrm{NMR}$, the vesicles were made in 90:10 (water/ $\left.\mathrm{D}_{2} \mathrm{O}\right), 100 \% \mathrm{D}_{2} \mathrm{O}$ for ${ }^{1} \mathrm{H}-$ NMR and $137 \mathrm{mM}$ PBS (pH 7.4) for dynamic light scattering (DLS), nano-differential scanning calorimetry (nano-DSC), and zeta potential experiments. The vesicles were prepared as described by Chen et al. (24). The samples were diluted to a lipid concentration of $1 \mathrm{mM}$ for DLS and zeta potential and $0.1 \mathrm{mM}$ for nano-DSC using $137 \mathrm{mM}$ PBS. Briefly, liposomes were prepared by dissolving $12.5 \mathrm{mg}$ of DPPC in $1 \mathrm{~mL}$ chloroform (for blank DPPC liposomes and GAR). Chloroform was removed by rotary evaporation at $50^{\circ} \mathrm{C}$ (above the DPPC melting temperature) starting at $450 \mathrm{mbar}$ for $30 \mathrm{~min}$, then decreased to $300 \mathrm{mbar}$ for $30 \mathrm{~min}$, and finally $200 \mathrm{mbar}$ for $30 \mathrm{~min}$. This lipid film was kept under vacuum for $12 \mathrm{~h}$ at room temperature in order to remove traces of chloroform. The film was then rehydrated with an appropriate aqueous medium (PBS for DLS, zeta potential, and DSC measurements; $100 \% \mathrm{D}_{2} \mathrm{O}$ for ${ }^{1} \mathrm{H}-\mathrm{NMR}$; and $10 \% \mathrm{D}_{2} \mathrm{O}$ for ${ }^{31} \mathrm{P}-\mathrm{NMR}$ ) for $2 \mathrm{~h}$ at $50^{\circ} \mathrm{C}$. Phenolic-containing liposomes were analogously prepared, with $17 \mathrm{mM}$ DPPC and $2 \mathrm{mM}$ of each phenolic compound dissolved in organic solvent and cast as a thin lipid film, followed by rehydration. QTN, RVR, or BPA along with DPPC were dissolved in methanol separately due to their limited solubility in chloroform, whereas for RAL liposomes, RAL and DPPC were dissolved in a 1:1 ratio of chloroform/ methanol due to the insolubility of RAL in pure chloroform or methanol. The organic solvents were removed by rotary evaporation at $50^{\circ} \mathrm{C}$ (above the DPPC melting temperature) starting at $450 \mathrm{mbar}$ for $30 \mathrm{~min}$, then decreased to $300 \mathrm{mbar}$ for $30 \mathrm{~min}$, and finally $200 \mathrm{mbar}$ for $30 \mathrm{~min}$. This lipid film was kept under vacuum for $12 \mathrm{~h}$ at room temperature to remove 
traces of organic solvents. The resulting thin films, containing $2 \mathrm{mM}$ hydrophobic phenolic for every $17 \mathrm{mM}$ DPPC, were then hydrated with the appropriate, aforementioned aqueous medium at $50^{\circ} \mathrm{C}$ for $2 \mathrm{~h}$. The resulting aqueous dispersions were then sonicated for $1 \mathrm{~h}$ using a bath sonicator maintained at $50^{\circ} \mathrm{C}$.

\section{Nuclear Magnetic Resonance}

All nuclear magnetic resonance (NMR) data were acquired on an Agilent NMRS $500 \mathrm{MHz}$ NMR spectrometer using a 5-mm NMRone probe, thermostated at $37^{\circ} \mathrm{C}$. Liposome formulations analyzed by NMR were prepared as previously described, with $10 \% v / v \mathrm{D}_{2} \mathrm{O}$ in water $\left({ }^{31} \mathrm{P}-\mathrm{NMR}\right)$ or $100 \% \mathrm{D}_{2} \mathrm{O}\left({ }^{1} \mathrm{H}-\mathrm{NMR}\right)$ used as the aqueous hydration medium in order to provide a deuterium lock signal. ${ }^{31} \mathrm{P}-\mathrm{NMR}$ data were collected at $202.3 \mathrm{MHz}$ for $60-\mathrm{K}$ scans with a $35.7-\mathrm{kHz}$ sweep width using $131-\mathrm{K}$ data points. Acquisition time was $1.3 \mathrm{~s}$ with a relaxation delay of $0.5 \mathrm{~s}$. A line broadening of $50 \mathrm{~Hz}$ was applied to all spectra. All spectra were indirectly referenced to $\mathrm{H}_{3} \mathrm{PO}_{4}$ set to $0 \mathrm{ppm}$. Data were acquired without spinning. ${ }^{1} \mathrm{H}-\mathrm{NMR}$ data were collected at $499.8 \mathrm{MHz}$ using the conditions defined in the figure captions. All NMR data were processed with Mnova program V8.1 Mestrelab Research, S.L.

\section{Nano-differential Scanning Calorimetry}

Nano-DSC was performed using a TA Instruments Nano DSC (New Castle, DE, USA). Samples at a concentration of $0.1 \mathrm{mM}$ lipid were degassed under vacuum for $30 \mathrm{~min}$ before loading into a $0.6-\mathrm{mL}$ capillary cell. The cell was then pressurized with nitrogen to $1 \mathrm{~atm}$ and equilibrated at $25^{\circ} \mathrm{C}$. The sample was scanned at $1^{\circ} \mathrm{C} \mathrm{min}^{-1}$ over a range of 10 to $60^{\circ} \mathrm{C}$.

\section{Dynamic Light Scattering}

DLS measurements were performed using a Malvern Instruments Zetasizer Nano ZS with a backscattering detector angle of $173^{\circ}$ and a 4-mW, 633-nm He-Ne laser (Worcestershire, UK). The samples were stored at $37^{\circ} \mathrm{C}$ and analyzed after 0,3 , and 5 days. For size distribution studies, $1-\mathrm{mL}$ aliquot parts of the liposome formulations were analyzed in an optical grade polystyrene cuvette at $37^{\circ} \mathrm{C}$.

\section{Zeta Potential}

An aliquot part of each formulation was diluted with $137 \mathrm{mM}$ PBS to give a final lipid concentration of $1 \mathrm{mM}$. Zeta potential values were then determined using a laser doppler procedure with a Malvern Instruments Zetasizer Nano ZS at $25^{\circ} \mathrm{C}$. Air drop interference was eliminated before measuring the zeta potential.

\section{RESULTS AND DISCUSSION}

The chemical structures of the phenolics studied in this investigation are shown in Fig. 1. The effects of the interactions between these compounds and DPPC molecules and their localization in the lipid bilayer on the surface characteristics and colloidal stability of DPPC liposomes are reported.

\section{Thermal Analysis}

Differential scanning calorimetry has been employed in order to determine the location of hydrophobic moieties in liposomal bilayers as well as appreciate the nature of their interaction with lipid molecules (25-27). The hydrophobic interior of a lipid bilayer is anisotropic in nature due to long range ordering of the acyl chains generated from cooperative interactions between the chains. A cooperative unit exists in the gel phase of a bilayer due to the transmission of motion among the fatty acid chains that are packed in a highly ordered hexagonal array (28). The transition from the gel to the liquid crystalline phase suggests a change from nearly all-trans to partially gauche conformation of $\mathrm{C}-\mathrm{C}$ bonds in the chains (29). This transition is an endothermic event and exhibits a specific profile in the DSC curve that provides information about the cooperative unit undergoing phase transition. Since the probability of $\mathrm{C}-\mathrm{C}$ gauche conformation is higher in the end region of the chain $\left(C_{10}\right.$ and higher), the center of a lipid bilayer tends to be more fluid due to disorder and the interactions in the $\mathrm{C}_{1}-\mathrm{C}_{10}$ region of the chain regulate the size of the cooperative unit undergoing phase transition. The location of a hydrophobic molecule in different regions of the bilayer (phosphorylcholine, glycerol backbone, $\mathrm{C}_{1}-\mathrm{C}_{10}$ methylene, and $\mathrm{C}_{10}$ and higher methylene) and its interaction with these regions may affect the cooperative unit and, consequently, the peak characteristics in the DSC profile. For instance, if a hydrophobic moiety is located in the $\mathrm{C}_{1}-\mathrm{C}_{10}$ methylene region, it interacts with the cooperative unit and broadens the phase transition peak whereas, if it is located closer to the center $\left(C_{10}\right.$ and higher methylene region) of the bilayer, the size of the cooperative unit is not affected and a sharper peak is detected. The packing of the cooperative unit can be altered in both cases, leading to an increase or decrease in the phase transition temperature, which is also referred to as the melting temperature $\left(\mathrm{T}_{\mathrm{m}}\right)$ of the bilayer. If a hydrophobic moiety interacts with the glycerol region of the bilayer, a shoulder peak is detected at a lower temperature along with the parent transition peak due to the formation of a new phase of its own smaller cooperative units that do not coexist with the parent cooperative unit of the bilayer. On the other hand, a completely new peak appears due to the formation of a new phase when chemical moieties such as cations and anions interact with the phosphorylcholine region of the bilayer (30).

As shown in Fig. 2, the liposomal bilayer of blank DPPC liposomes displayed a typical sharp peak at about $43^{\circ} \mathrm{C}$ due to transition from a gel to a liquid crystalline phase. The effect of the various hydrophobic phenolics incorporated into DPPC liposomes is also depicted in their respective DSC thermograms. It can be inferred that RAL was located in the $\mathrm{C}_{1}-\mathrm{C}_{10}$ methylene region of the bilayer and interacted with the parent cooperative unit, since the phase transition peak was broadened after incorporation of RAL into the bilayer. The entropy of this phase transition (Table I) appeared to slightly increase, and the enthalpy appeared to increase due to an apparent increase in the $T_{m}$ to about $49^{\circ} \mathrm{C}$. Such an increase in the $T_{m}$ due to the incorporation of RAL suggests that the packing of the cooperative unit was altered in such a way as to enhance the rigidity of the bilayer. Like RAL, GAR also exhibited a peak broadening effect, suggesting its incorporation into the 


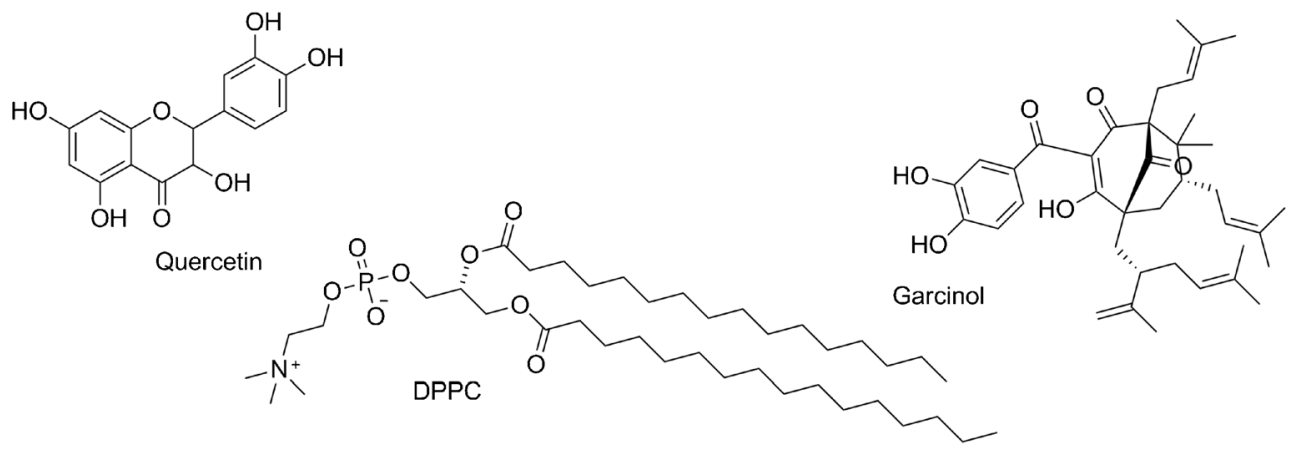<smiles>[R]OC(=O)c1ccc(O)cc1</smiles><smiles></smiles><smiles>CC(CC(Br)(Br)Br)(c1ccc(O)cc1)c1ccc(O)cc1</smiles>

Fig. 1. Chemical structures of phenolic compounds and DPPC

$\mathrm{C}_{1}-\mathrm{C}_{10}$ methylene region. In the case of GAR, however, the entropy, enthalpy, and the $T_{m}$ apparently decreased as compared to blank DPPC control liposomes, suggesting a slight reduction in the bilayer rigidity. A slight reduction in the $T_{m}$, broadening of the transition peak, and the appearance of a small shoulder at the lower temperature suggests that BPA was located in between the glycerol and the $\mathrm{C}_{1}-\mathrm{C}_{10}$ methylene regions and strongly interacted with the glycerol groups of DPPC molecules. Although the incorporation of RVR and QTN also depicted a slight reduction in the $T_{m}$, no peak broadening was detected, suggesting that the location of these drug molecules was closer to the center $\left(\mathrm{C}_{10}-\mathrm{C}_{16}\right.$ methylene region) of the bilayer. The broad peak at $43^{\circ} \mathrm{C}$ suggests nonisotropic transfer of heat across the liposome as all the chains are not arranged in a uniform array. There is distortion in the $\mathrm{C}_{10}-\mathrm{C}_{16}$ region due to gauche conformation as opposed to trans in the upper region, i.e., $\mathrm{C}_{1}-\mathrm{C}_{10}$. The peak sharpening could be attributed to the presence of RVR and QTN in the $\mathrm{C}_{10}-\mathrm{C}_{16}$ region and thereby assisting in a more uniform heat transfer by arranging the lipid chains in an ordered array. Since the concentration of all of the phenolic compounds that were used in this work was relatively high $(2 \mathrm{mM})$, and since their aqueous solubility is very low as compared to their affinity for lipids, a natural consequence of minimizing unfavorable hydrophobic interactions with water while optimizing hydrogen bond interactions and free energy within the bilayer, it might be inferred that the center region of the bilayer could have been saturated with the drug in the case of both RVR and QTN. Consequently, this may have increased the cooperativity of phase transition leading to a sharpening of the transition peak and an increase in the entropy and enthalpy of phase transition.

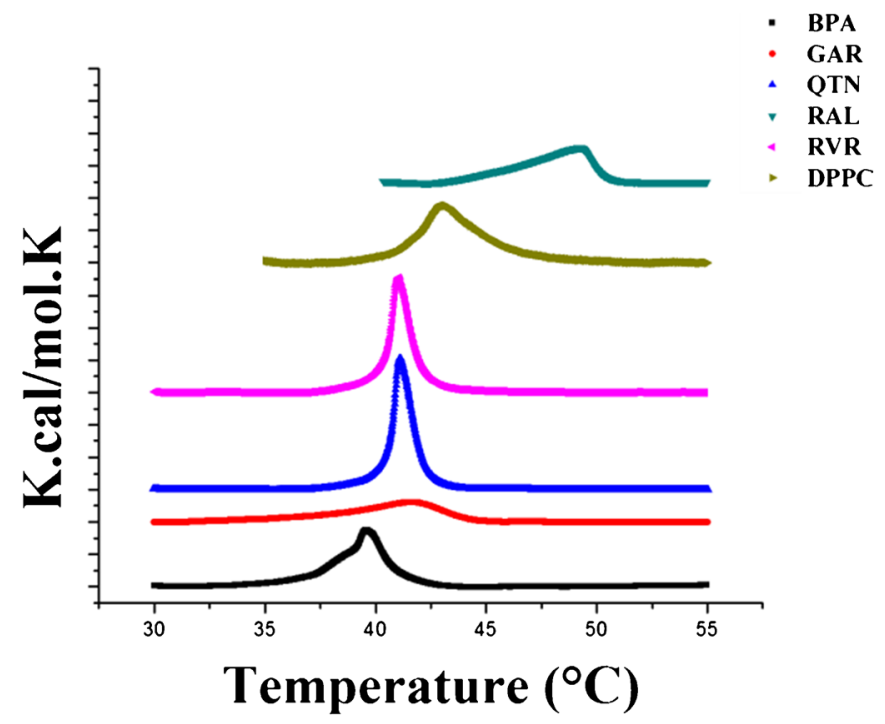

Fig. 2. DSC endotherms depicting the effect of phenolic compounds on the $T_{m}$ of DPPC liposomes 
Table I. Enthalpy, Entropy, and Transition Temperatures of Different Liposomal Formulations Containing Diphenolic and Polyphenolic Analytes

\begin{tabular}{lccc}
\hline Formulations & $\Delta \mathrm{H}(\mathrm{Kcal} / \mathrm{mol})$ & $\begin{array}{c}\Delta \mathrm{S}[\mathrm{Kcal} / \\
(\mathrm{mol} . \mathrm{K})\end{array}$ & $\mathrm{T}_{\mathrm{m}}\left({ }^{\circ} \mathrm{C}\right)$ \\
\hline Blank DPPC & 08.43 & 0.1963 & 42.96 \\
DPPC + 2 mM RAL & 12.12 & 0.2463 & 49.24 \\
DPPC + 2 mM RVR & 13.30 & 0.3242 & 41.05 \\
DPPC + 2 mM QTN & 14.66 & 0.3565 & 41.12 \\
DPPC + 2 mM BPA & 11.68 & 0.2952 & 39.58 \\
DPPC + 2 mM GAR & 07.98 & 0.1917 & 41.62 \\
\hline
\end{tabular}

$D P P C$ dipalmitoylphosphatidylcholine, $R A L$ raloxifene, $R V R$ transresveratrol, $Q T N$ quercetin, $B P A$ bisphenol A, GAR garcinol

\section{${ }^{31}$ P-NMR}

The incorporation and localization of RVR, RAL, QTN, BPA, and GAR into DPPC liposomes were assessed by ${ }^{31} \mathrm{P}-$ NMR, as shown in Fig. 3. In the case of blank DPPC liposomes, one single sharp peak was obtained, which is characteristic of small, unilamellar vesicles (28). The presence of small, unilamellar vesicles indicated by NMR and DSC data was confirmed in representative formulations using cryo-TEM imaging (data not shown; Supplemental 1). In QTNcontaining DPPC liposomes, no chemical shift was observed, but the line shape broadened slightly. This peak broadening was unlikely to be caused by larger vesicles in solution because of reproducible liposome preparation which yielded liposomes very similar in terms of size and morphology (as indicated by DLS and cryo-TEM data). It appeared that there was a dynamic process involved with the phosphate head groups in the presence of QTN. However, the DSC data suggest that QTN was located closer to the center of the bilayer, a situation that influenced the orderliness of the acyl chains as it sharpened the transition peak. The NMR peak broadening is perhaps an indirect effect on the change in the environment of the phosphate head groups due to an increase in the orderliness of the acyl chains, as suggested by the shape of the corresponding DSC curve. In both RAL- and BPA-containing DPPC liposomes, an additional resonance appeared upfield of the DPPC resonance due to shielding and increased surface exposure of the phosphate groups. This upfield resonance was likely a result of additional shielding, perhaps induced by the rigid aromatic rings of RAL and BPA, which may have oriented themselves in the lipid bilayer in the proximity of the phosphate head groups. In addition, BPA incorporation resulted in a shoulder at a lower temperature in the DSC curve and a peak broadening effect similar to RAL, suggesting localization of both drugs in the vicinity of the glycerol moiety in the $\mathrm{C}_{1}-$ $\mathrm{C}_{10}$ chain region. GAR showed a similar effect with a much broader peak. This might be attributed to multiple stable interactions with the phosphate head groups and their enhanced surface exposure. RVR showed the broadest peak, which again suggests multiple stable interactions with the head group. However, RVR, like QTN, was presumed to be present closer to the center of the bilayer in the $\mathrm{C}_{10}-\mathrm{C}_{16}$ region. While it is theoretically possible that RVR and QTN could be well mixed throughout the bilayers, no aromatic (from drug) to aliphatic (N-methyl moieties of the choline region) NOEs were observed, further suggesting localization in the $\mathrm{C}_{10}-\mathrm{C}_{16}$ region, a postulate supported by ${ }^{31} \mathrm{P}-\mathrm{NMR}$, which revealed a lack of any shielding effect. Thus, there was no direct correlation between the DSC data and the NMR peak broadening, which might be due to an RVR-induced increase in the orderliness of the bilayer that in turn affected the phosphate head group environment, possibly reducing their surface exposure.

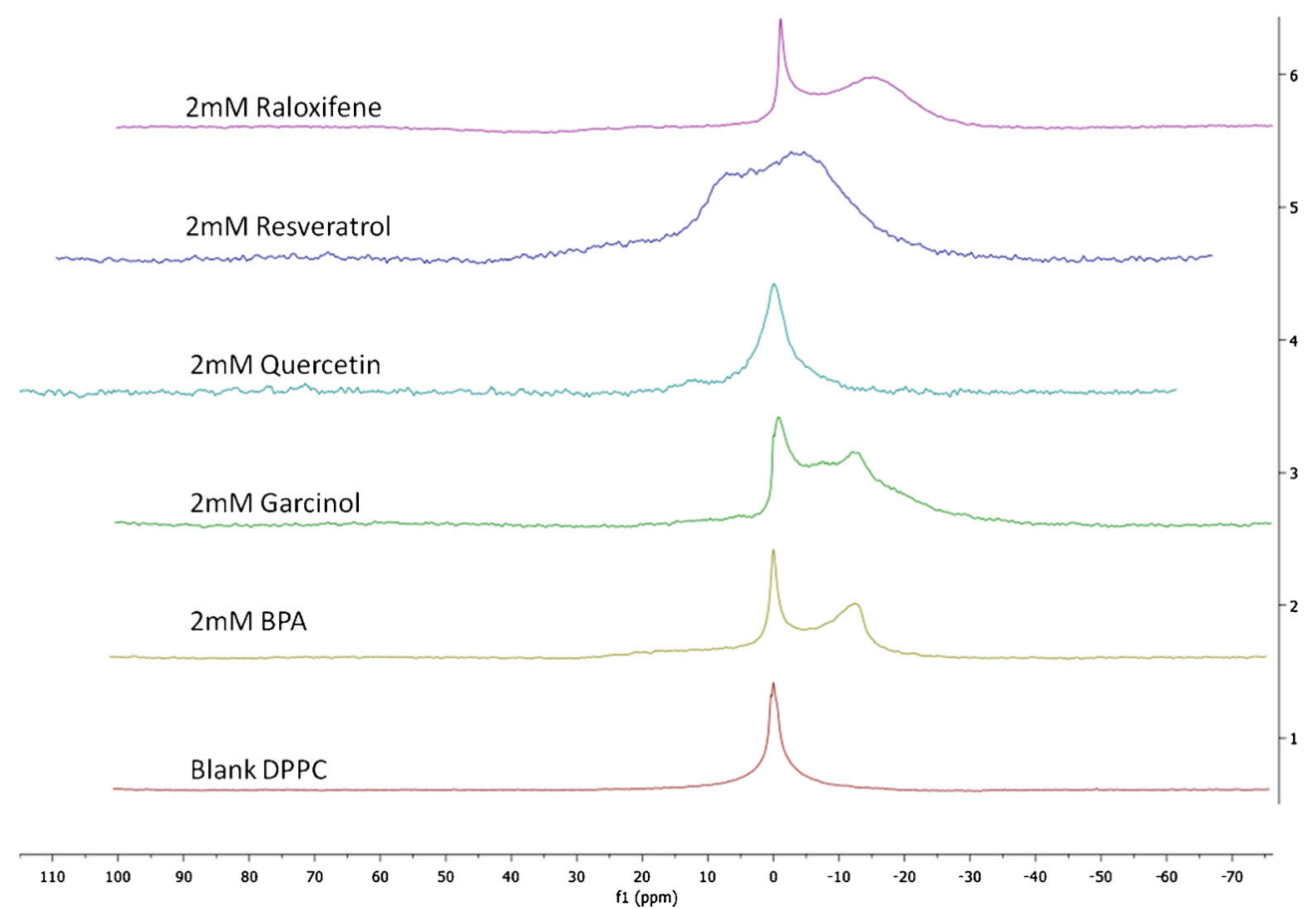

Fig. 3. ${ }^{31} \mathrm{P}-\mathrm{NMR}$ spectra after $60-\mathrm{K}$ scans at $37^{\circ} \mathrm{C}$ in $(9: 1)$ water $/ \mathrm{D}_{2} \mathrm{O}$ as solvent, characterizing the interactions of the phenolic analytes with the phosphate head groups of DPPC liposomes 


\section{${ }^{1}$ H-NMR}

As shown in Fig. 4, all resonances in the blank DPPC liposome spectrum were broadened, a result characteristic of the formation of unilamellar vesicles. These results are consistent with general NMR principles and previous observations of vesicles $(31,32)$. The phospholipid glycerol resonances $(5.1 \mathrm{ppm})$ were broadened almost to the point where they disappeared, indicating minimal local molecular motions for these protons. The glycerol methine resonance sharpened and became clearly observable in liposomes loaded with BPA. The decreased line width was obtained because of an increase in local molecular motions in that region of the phospholipid. This would suggest that BPA was bound near the head group region. Also observed were the aromatic resonances of BPA in the NMR spectrum at $7.12 \mathrm{ppm}(2,6$ and $\left.2^{\prime}, 6^{\prime}\right)$ and at $6.84 \mathrm{ppm}\left(3,5\right.$ and $\left.3^{\prime}, 5^{\prime}\right)$. All of the aromatic resonances were broadened, which is indicative of the entrapment of BPA in the hydrophobic region of the vesicle bilayer (33). The BPA methyl resonance was not discernible in the spectrum because of the overlapping DPPC resonances. In the case of RAL, vesicle resonances were similar to those observed for BPA-loaded vesicles. Aromatic RAL resonances were noted with broadened line widths indicative of incorporation into the vesicles. The DPPC resonances were sharper in RAL-containing liposomes as compared with those observed in blank DPPC liposome controls. The observed aromatic protons integrated to more than the expected value for one species of RAL. Assigning a value of one proton to the smallest aromatic resonance gave a total proton count of approximately 22, which is double the expected proton count for RAL. A possible explanation for this observation is that RAL was bound to the vesicle in two different ways which did not interconvert on the NMR time scale. From Fig. 5a, more than the expected number of aromatic to aromatic NOEs in the 2D NOESY of RAL-containing liposomes were observed. In bulk solvent, RAL would be expected to exhibit three aromatic to aromatic NOEs. Two NOEs would arise from the protons of the two para-substituted phenyl rings and would be twice the intensity of the NOE between the orthoprotons of the benzothiophene ring of RAL. In RALcontaining liposomes, NOEs for five aromatic to aromatic interactions were noted, and while these interactions have not been assigned, it would seem unlikely that they were all intramolecular NOEs, and some of the cross-peak may represent exchange between multiple orientations of RAL in the vesicle bilayer environment. Also observed were NOEs from two of the aromatic resonances to the $N$-methyl resonance of the choline head group (indicated by the black arrow). In Fig. 5b, additional NOEs are observed between the BPA resonance at $6.84 \mathrm{ppm}$ and the $-\mathrm{N}(\mathrm{CH})_{3}$ of the choline and a methylene near the carbonyl moiety of the acyl chains. This further supports the localization of BPA near the glycerol region. A single, broad aromatic resonance was observed in both the 1D and 2D data collected for RVR (data not shown; Supplemental 2). No resonances from GAR were observed in either 1D or 2D NMR data (data not shown; Supplemental 2). These results suggest that GAR and RVR bound very tightly within the vesicle bilayer. Evidence of this binding was seen because the $-\mathrm{CH}_{2}$ and $-\mathrm{CH}_{3}$ resonances from DPPC were sharper in GAR- and RVR-containing liposomes as compared to blank DPPC controls. This effect was similar to the other polyphenolics used in the study. In the case of QTN, five broadened aromatic peaks consistent with the QTN structure were observed (Fig. 6).

Again, the peak broadening suggests QTN binding to the vesicle bilayer. Also seen were changes in the DPPC spectrum indicative of QTN binding. The $-\mathrm{CH}_{2}$ and $-\mathrm{CH}_{3}$ peaks were

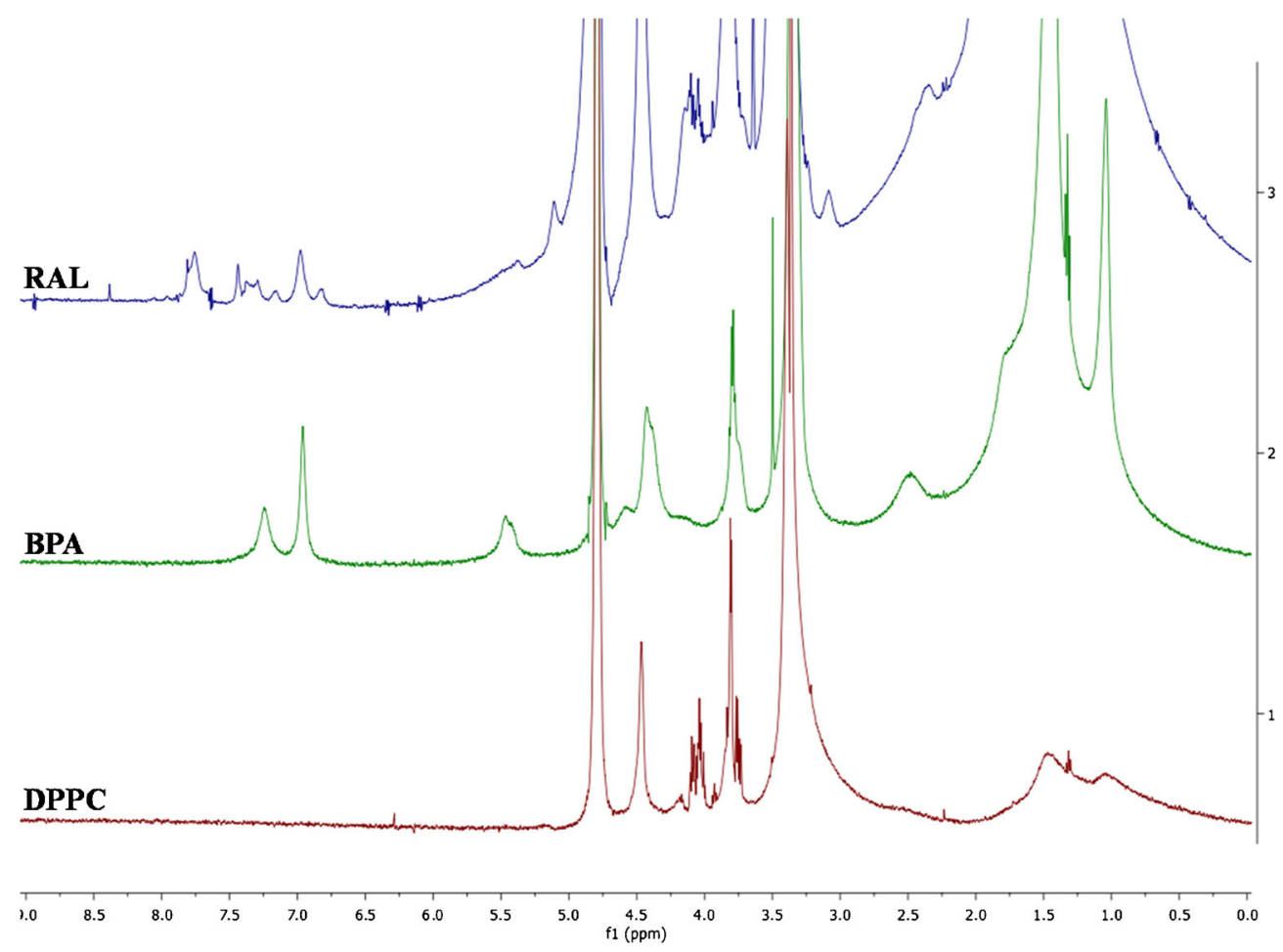

Fig. 4. $1 \mathrm{D}{ }^{1} \mathrm{H}-\mathrm{NMR}$ spectra of RAL, BPA, and blank DPPC liposomes showing aromatic peaks from the phenolic molecules 

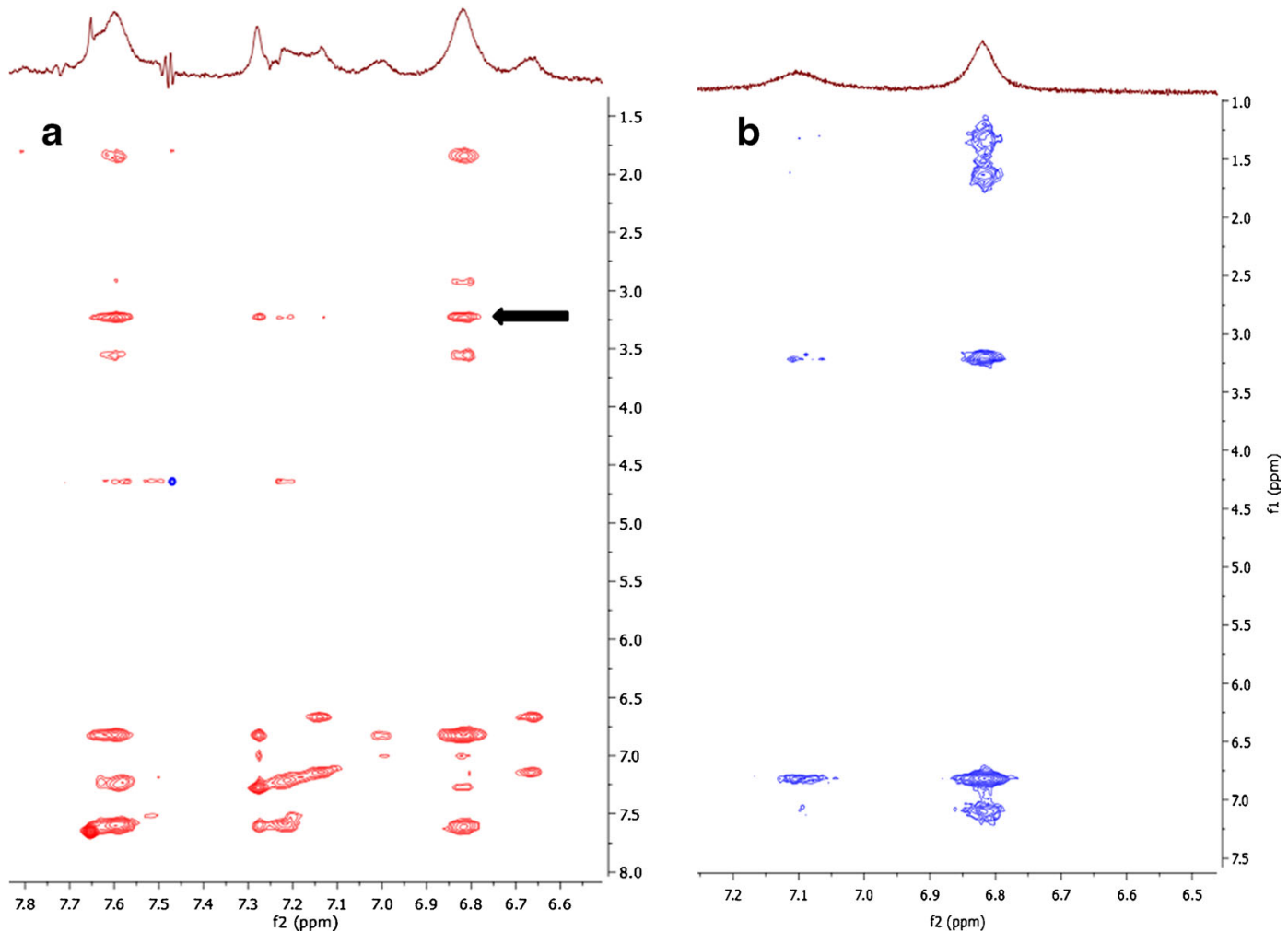

Fig. 5. $2 \mathrm{D}{ }^{1} \mathrm{H}-\mathrm{NMR}$ spectra of a RAL- and $\mathbf{b}$ BPA-containing DPPC liposomes

sharpened, as was observed with other phenolics assessed in this study (Fig. 4). Also seen was a sharpened glycerol methine resonance as further proof of binding. The aromatic peaks observed were very broad. It was hypothesized that above the $T_{m}$ of these vesicles, the molecular motion of QTN in the vesicle bilayer would increase, which could be

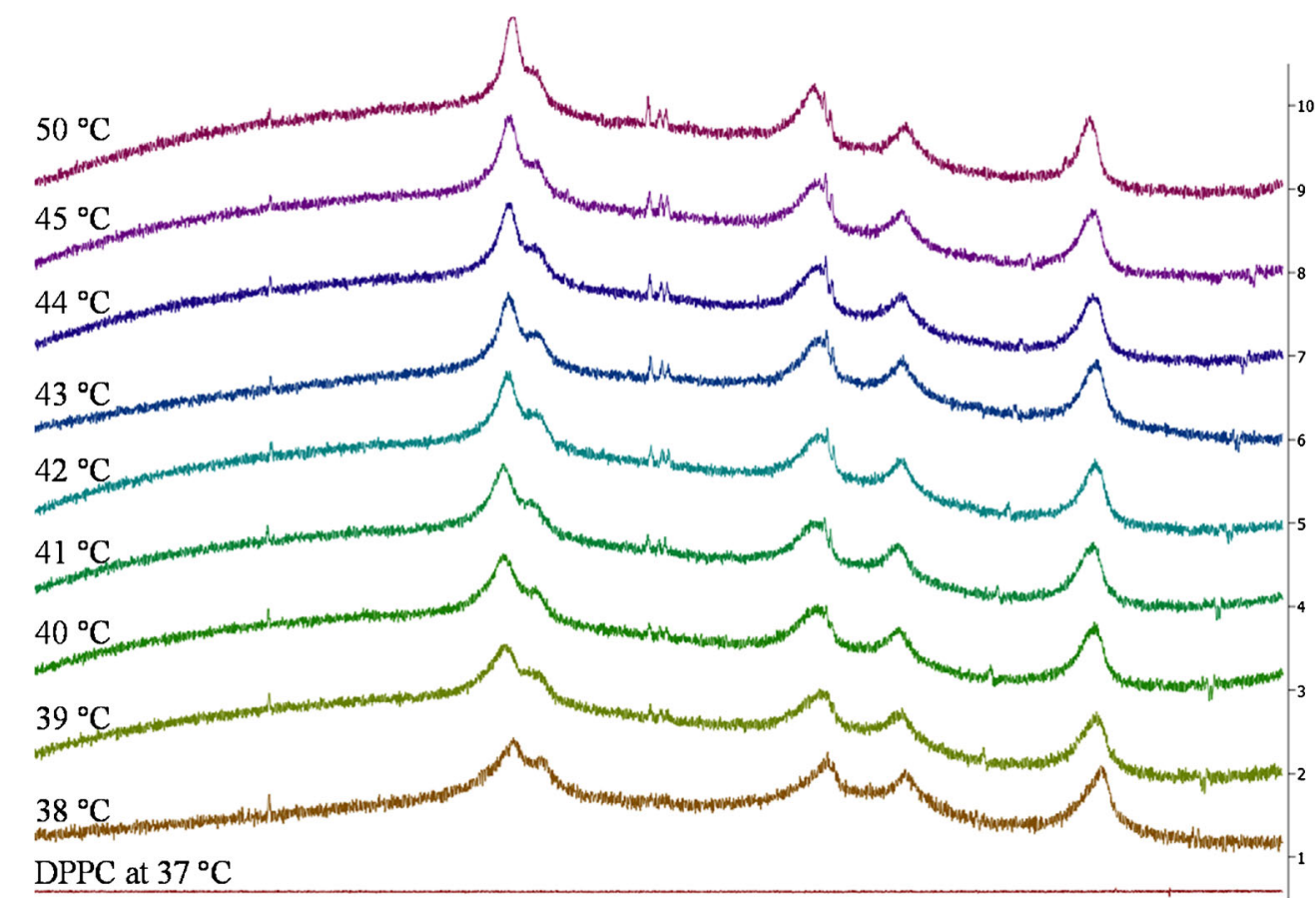

$\begin{array}{lllllllllllllllllllllllllllllllllllllllllllllll}9.0 & 8.9 & 8.8 & 8.7 & 8.6 & 8.5 & 8.4 & 8.3 & 8.2 & 8.1 & 8.0 & 7.9 & 7.8 & 7.7 & 7.6 & 7.5 & 7.4 & 7.3 & 7.2 & 7.1 & 7.0 & 6.9 & 6.8 & 6.7 & 6.6 & 6.5 & 6.4 & 6.3 & 6.2 & 6.1 & 6.0 & 5.9 & 5.8 & 5.7\end{array}$

Fig. 6. $1 \mathrm{D}{ }^{1} \mathrm{H}-\mathrm{NMR}$ spectra of (1) blank and (2-10) QTN-containing DPPC liposomes. No aromatic peaks were seen in blank DPPC liposomes whereas QTN peaks sharpened with the application of heat due to increased molecular mobility 
indicated by sharper peaks. Thus, the QTN-containing liposomes were heated from 38 to $45^{\circ} \mathrm{C}$ (the $\mathrm{T}_{\mathrm{m}}$ of QTNcontaining liposome was $41.12^{\circ} \mathrm{C}$ ) at $1^{\circ} \mathrm{C}$ increments $(10 \mathrm{~min}$ temperature equilibration time) and 1D NMR spectra were then collected after each temperature increment (Fig. 6). Throughout the experiment, there was no significant chemical shift change for QTN resonances, indicating a consistent molecular environment. All five QTN resonances sharpened in appearance as the temperature increased, a phenomenon indicative of increased molecular motion in the bilayer. As the temperature increased, sharp resonances (ca. 7.35-7.45 ppm) consistent with QTN in bulk $\mathrm{D}_{2} \mathrm{O}$ were also observed. The intensity of these resonances increased with increasing temperature until $41^{\circ} \mathrm{C}$, suggesting release of QTN from the vesicle bilayer into bulk solvent. Between 41 and $50^{\circ} \mathrm{C}$, the intensities remained constant, suggesting saturation of QTN in the bulk $\mathrm{D}_{2} \mathrm{O}$.

\section{Particle Size and Zeta Potential Analysis}

The particle size analysis was conducted by DLS in PBS in triplicate over serial time intervals until aggregation was detected after 5 days. As seen in Fig. 7, several formulations maintained relatively consistent particle size except those containing QTN and RVR. It is evident that, despite their effect on increasing the orderliness of the acyl chains of DPPC, QTN- and RVR-containing liposomes were relatively unstable and underwent aggregation over time. Thus, it could be inferred that the location of these and perhaps other hydrophobic compounds closer to the center of the bilayer may adversely affect the colloidal stability of the liposomes due to reduced surface exposure of the phosphate head groups. In contrast, RAL, GAR, and BPA, located in the vicinity of the head group or $\mathrm{C}_{1}-\mathrm{C}_{10}$ chain segment of DPPC, did not adversely affect the colloidal stability of liposomes, as was evident from particle size data, presented as $z$-average intensity measurements, with polydispersity indices (PDI) for DPPC controls and liposomes containing RAL, GAR, BPA, QTN, and RVR of $0.07,0.056,0.233,0.120,0.043$, and 0.234,

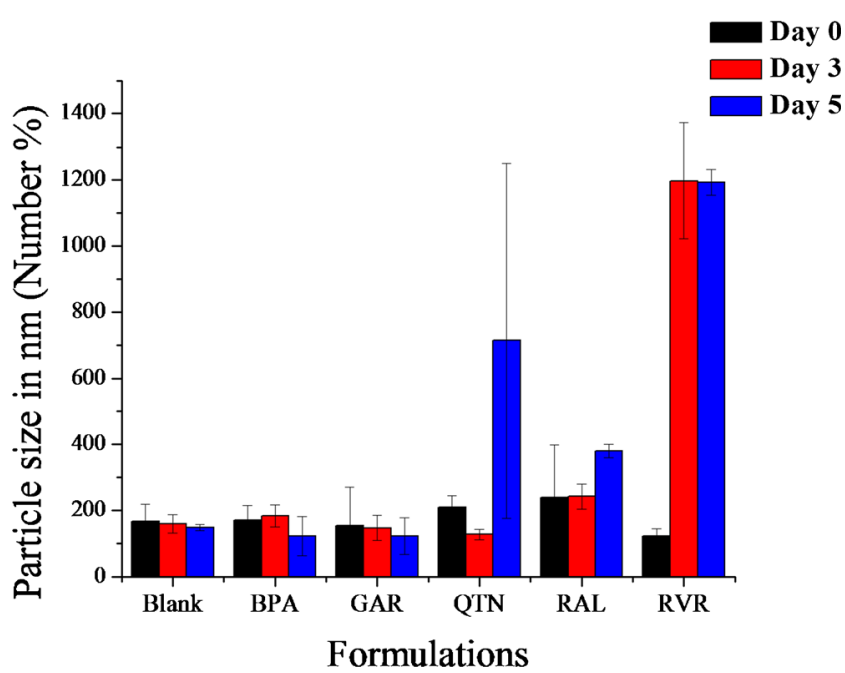

Fig. 7. Liposome size (in $\mathrm{nm}$ ) measured by DLS at $37^{\circ} \mathrm{C}$ over a period of 5 days, indicating aggregation of QTN- and RVR-containing DPPC liposomes respectively. It can be hypothesized that hydrophobic molecules located in the vicinity of the glycerol and $\mathrm{C}_{1}-\mathrm{C}_{10}$ acyl chain regions and interacting with the lipid molecules may affect phosphate head group orientation (as demonstrated by ${ }^{31} \mathrm{P}-\mathrm{NMR}$ spectroscopy) in such a way as to restrict aggregation and/or fusion of liposomes, perhaps by enhancing surface exposure, electrostatic repulsion, and inducing steric hindrance. In contrast, molecules located closer to the center of the bilayer from $\mathrm{C}_{10}$ and higher may reduce the surface exposure of phosphate head groups, leading to colloidal instability.

Zeta potential measurement was used to quantify the extent of liposomal phosphate group surface exposure in the presence of the phenolic compounds by measuring the surface charge. The zeta potential of blank DPPC liposomes over a period of 5 days was $-2 \pm 1 \mathrm{meV}$, which is consistent with the literature and is attributed to the adsorption of $\mathrm{OH}^{-}$ions from the surrounding buffer and to surface exposure of the phosphate head groups $(34,35)$. The zeta potential of GARcontaining liposomes was a constant $-11 \pm 2 \mathrm{meV}$ over a period of 5 days, which could be attributed to the localization of the large GAR molecules in the vicinity of the head groups and a subsequent increase in the surface exposure of phosphate head groups at the interface. These data correlate with the DSC peak broadening effect of GAR due to a decrease in the cooperativity of mixing and its localization at the $\mathrm{C}_{1}-\mathrm{C}_{10}$ region of the DPPC acyl chains. In contrast, QTN, RVR, RAL, and BPA had no significant effect on the zeta potential of the liposomes. The zeta potential of these formulations was $-1 \pm 2 \mathrm{meV}$ for a period of 5 days and was consistent over the course of the experiment. Since QTN and RVR were present closer to the center of the bilayer and did not lead to increased phosphate group exposure, the zeta potential of QTN- and RVR-containing liposomes was not altered. As demonstrated by ${ }^{31}$ P-NMR, RAL and BPA were located closer to the phosphate groups in the bilayer as compared to RVR and QTN and resulted in increased phosphate group exposure. Nevertheless, the zeta potential was not changed in RAL- and BPAcontaining liposomes, perhaps due to their less bulky, less constrained structure and a more widely separated, linear orientation of phenolic groups as compared to GAR. As compared to GAR, RAL and BPA may have strongly influenced phosphate group exposure and may also have caused less adsorption of $\mathrm{OH}^{-}$ions from the surrounding buffer.

\section{CONCLUSIONS}

The location of hydrophobic phenolics in DPPC liposomal bilayers was determined using a variety of complementary analytical techniques. The incorporation of hydrophobic phenolics into liposomal bilayers may profoundly affect bilayer organization, surface characteristics, and vesicle stability based upon their localization in various regions of phospholipid bilayers. Despite certain similar physiochemical properties, these phenolics vary in geometry, flexibility, and molecular weight and thus may interact differently with the lipid molecules and may be located in different regions or stably located in multiple regions, as seen in the case of RAL, throughout the phospholipid bilayer. While the specific orientation and configuration of the phenolic molecules within the bilayer were undetermined, the phenolic compounds that were shown to be 
located closer to the center of the bilayer adversely affected the colloidal stability of the liposomes. These data may be useful in the design of liposomal systems for the encapsulation and formulation of hydrophobic phenols and other small molecules. Likewise, they may provide guidance for the selection of appropriate analytical methods for determining the location of small molecules within liposomal bilayers as well as for predicting compound localization in lipid bilayers and their subsequent effects on liposomal stability.

\section{ACKNOWLEDGMENTS}

This material is based upon work conducted at a research facility at the University of Rhode Island supported in part by the National Science Foundation EPSCoR Cooperative Agreement no. EPS-1004057.

\section{REFERENCES}

1. Edwards KA, Baeumner AJ. Talanta. 2006;68(5):1421-31.

2. Bangham AD, Standish MM, Watkins JC. J Mol Biol. 1965;13(1):238-52.

3. Immordino ML, Dosio F, Cattel L. Int J Nanomedicine. 2006;1(3):297-315.

4. Gulati $\mathbf{M}$, Grover $\mathrm{M}$, Singh $\mathrm{S}$, Singh $\mathrm{M}$. Int J Pharm 1998;165:129-68.

5. Coimbra M, Isacchi B, van Bloois L, Torano JS, Ket A, Wu X, et al. Int J Pharm. 2011;416(2):433-42.

6. Liang X, Mao G, Ng KY. J Colloid Interface Sci. 2005;285(1):360-72.

7. Lee H, Kim HR, Park JC. Phys Chem Chem Phys. 2014;16(8):3763-70.

8. Cai D, Gao W, He B, Dai W, Zhang H, Wang X, et al. Biomaterials. 2014;35(7):2283-94.

9. Pawlikowska-Pawlega B, Misiak LE, Zarzyka B, Paduch R, Gawron A, Gruszecki WI. Biochim Biophys Acta. 2013;1828(2):518-27.

10. Wu RG, Dai JD, Wu FG, Zhang XH, Li WH, Wang YR. Int J Pharm. 2012;438(1-2):91-7.

11. Notman R, Noro MG, Anwar J. J Phys Chem B. 2007;111(44):1274855.
12. Pawlikowska-Pawlega B, Misiak LE, Zarzyka B, Paduch R, Gawron A, Gruszecki WI. Biochim Biophys Acta. 2012;1818(7):1785-93.

13. Huh NW, Porter NA, McIntosh TJ, Simon SA. Biophys J. 1996;71(6):3261-77.

14. Olas B, Holmsen H. Food Chem Toxicol. 2012;50(11):4028-34.

15. Bonechi C, Martini S, Ciani L, Lamponi S, Rebmann H, Rossi C, et al. PLoS ONE. 2012;7(8):e41438.

16. Masullo M, Menegazzi M, Di Micco S, Beffy P, Bifulco G, Dal Bosco M, Novelli M, Pizza C, Masiello P, Piacente S. J Nat Prod. 2013.

17. Bakana P, Claeys M, Totte J, Pieters LA, Van Hoof L, Tamba V, et al. J Ethnopharmacol. 1987;21(1):75-84.

18. Cadena PG, Pereira MA, Cordeiro RB, Cavalcanti IM, Barros Neto B, Pimentel Mdo C, et al. Biochim Biophys Acta. 2013;1828(2):309-16.

19. Spedding G, Ratty A, Middleton Jr E. Antivir Res. 1989;12(2):99-110.

20. Park HJ, Lee CM, Jung ID, Lee JS, Jeong YI, Chang JH, et al. Int Immunopharmacol. 2009;9(3):261-7.

21. Nothlings U, Murphy SP, Wilkens LR, Henderson BE, Kolonel LN. Am J Epidemiol. 2007;166(8):924-31.

22. Jung MK, Hur DY, Song SB, Park Y, Kim TS, Bang SI, et al. J Invest Dermatol. 2010;130(5):1459-63.

23. Delclos KB, Camacho L, Lewis SM, Vanlandingham MM, Latendresse JR, Olson GR, Davis KJ, Patton RE, da Costa GG, Woodling KA, Bryant MS, Chidambaram M, Trbojevich R, Juliar BE, Felton RP, Thorn BT. Toxicol Sci. 2014.

24. Chen Y, Bose A, Bothun GD. ACS Nano. 2010;4(6):3215-21.

25. Joanne P, Galanth C, Goasdoue N, Nicolas P, Sagan S, Lavielle S, et al. Biochim Biophys Acta. 2009;1788(9):1772-81.

26. Ikeda A, Kiguchi K, Shigematsu T, Nobusawa K, Kikuchi J, Akiyama M. Chem Commun (Camb). 2011;47(44):12095-7.

27. El Maghraby GM, Williams AC, Barry BW. Int J Pharm. 2005;292(1-2):179-85.

28. Alaouie AM, Smirnov AI. Biophys J. 2005;88(2):L11-3.

29. Wang G, Lin HN, Li S, Huang CH. J Biol Chem. 1995;270(39):22738-46.

30. Jain M, Wu NM. J Membr Biol. 1976;34:157-201.

31. Romberg B, Kettenes-van den Bosch JJ, de Vringer T, Storm G, Hennink WE. Bioconjug Chem. 2006;17(3):860-4.

32. Pawlikowska-Pawlega B, Dziubinska H, Krol E, Trebacz K, Jarosz-Wilkolazka A, Paduch R, et al. Biochim Biophys Acta. 2013;1838(1):254-65.

33. Pawar B, Joshi M, Srivastava S, Kanyalkar M. J Pharm Pharmacol. 2012;64(6):802-10.

34. Cevc G. Chem Phys Lipids. 1993;64:163-86.

35. Cullis PR, de Kruijff B. Biochim Biophys Acta. 1979;559(4):399420. 